\title{
The OBRA-87 Nursing Home Regulations and Implementation of the Resident Assessment Instrument: Effects on Process Quality
}

\author{
Catherine Hawes, PhD, Vincent Mor, PhD, ${ }^{\dagger}$ Charles D. Phillips, PhD, MPH, ${ }^{\ddagger}$ \\ Brant E. Fries, PhD, John N. Morris, PhD, I Eliana Steele-Friedlob,"Angela M. Greene," and \\ Marianne Nennstiel"
}

OBJECTIVE: To characterize changes in key aspects of process quality received by nursing home residents before and after the implementation of the national nursing home Resident Assessment Instrument (RAI) and other aspects of the Omnibus Budget Reconciliation Act (OBRA) nursing home reforms.

DESIGN: A quasi-experimental study using a complex, multistage probability-based sample design, with data collected before (1990) and after (1993) implementation of the RAI and other OBRA provisions.

SETTING AND PARTICIPANTS: Two independent cohorts ( $\mathrm{n}>2000)$ of residents in a random sample of 254 nursing facilities located in metropolitan statistical areas in 10 states.

INTERVENTION: OBRA-87 enhanced the regulation of nursing homes and included new requirements on quality of care, resident assessment, care planning, and the use of neuroleptic drugs and physical restraints. One of the key provisions, used to help implement the OBRA requirements in daily nursing home practice, was the mandatory use of a standardized, comprehensive system, known as the RAI, to assist in assessment and care planning. OBRA provisions went into effect in federal law on October 1, 1990, although delays issuing the regulations led to actual implementation of the RAI during the Spring of 1991.

MEASUREMENTS AND ANALYSES: Research nurses spent an average of 4 days per facility in each data collection round, assessing a sample of residents, collecting data through interviews with and observations of residents, interviews with multiple shifts of direct staff caregivers for the sampled residents, and review of medical records, including physician's orders, treatment and care plans, nursing progress notes, and medication records. The RNs collected data on the characteristics of the

From the "Research Triangle Institute, Research Triangle Park, North Carolina; tDepartment of Community Health and Center for Gerontology and Health Care Research, Brown University, Providence, Rhode Island; † Myers Research Institute, Menorah Park Center on Aging, Beachwood, Ohio; \$Institute of Gerontology and School of Public Health, University of Michigan and VA Medical Center, Ann Arbor, Michigan; HRCA Research and Training Institure, Hebrew Rehabilitation Center for Aged, Boston, Massachusetts; and IMEDSTAT Group, Atlanta Georgia.

The work of the authors on this paper was supported, in part, by contract DHHS 500-88-0055 from the Health Care Financing Administration, Health Standards and Quality Bureau.

Address correspondence and reprint requests to Catherinc Hawes, PhD, Research Triangle Institute, 8450 Whispering Pines, Novelty, $\mathrm{OH} 44072$. sampled residents, on the care they received, and on facility practices. The effect of being a member of the 1990 pre-OBRA or the 1993 post-OBRA cohort was assessed on the accuracy of information in the residents' medical records, the comprehensiveness of care plans, and on other key aspects of process quality while controlling for any changes in resident case-mix. The data were analyzed using contingency tables and logistic regression and a special statistical software (SUDAAN) to assure proper variance estimation.

RESULTS: Overall, the process of care in nursing homes improved in several important areas. The accuracy of information in residents' medical records increased substantially, as did the comprehensiveness of care plans. In addition, several problematic care practices declined during this period, including use of physical restraints (37.4 to $28.1 \%(P<$ $.001))$ and indwelling urinary catheters $(9.8$ to $7 \%(P<$ $.001))$. There were also increases in good care practices, such

\section{For editorial comment, see pp 975, 1025, and 1027}

as the presence of advanced directives, participation in activities, and use of toileting programs for residents with bowel incontinence. These results were sustained after controlling for differences in the resident characteristics between 1990 and 1993. Other practices, such as use of antipsychotic drugs, behavior management programs, preventive skin care, and provision of therapies were unaffected, or the differences were not statistically significant, after adjusting for changes in resident case-mix. CONCLUSION: The OBRA reforms and introduction of the RAI constituted an unprecedented implementation of comprehensive geriatric assessment in Medicare- and Medicaidcertified nursing homes. The evaluation of the effects of these interventions demonstrates significant improvements in the quality of care provided to residents. At the same time, these findings suggest that more needs to be done to improve process quality. The results suggest the RAI is one tool that facility staff, therapists, pharmacy consultants, and physicians can use to support their continuing efforts to provide high quality of care and life to the nation's 1.7 million nursing home residents. J Am Geriatr Soc 45:977-985, 1997.

JAGS $45: 977-985,1997$

(1) 1997 by the American (ieriatrics Society 
Tn the fall of 1990 , nursing homes that participated in the Medicare or Medicaid programs began operating under a new set of federal regulations mandated by Congress in the Omnibus Budget Reconciliation Act of 1987 (OBRA). These reforms were the product of decades of scandals about inadequate care and ineffective regulation, a federal class action law suit, ${ }^{1}$ and a Congressionally mandated study of nursing home regulation conducted by the Institute of Medicine. ${ }^{2}$ The OBRA reforms specified comprehensive changes in the way nursing homes were regulated, including new standards of care, a resident-focused, outcome-oriented survey process, and a range of new federal enforcement remedies. ${ }^{3}$ The new standards required that Medicare- and Medicaid-certified nursing facilities use a standardized, "reproducible," comprehensive functional assessment tool to assess all residents and guide the development of individualized care plans. This instrument, the Resident Assessment Instrument (RAI), was developed under contract with the Health Care Financing Administration (HCFA). ${ }^{4}$

The Minimum Data Set for Nursing Home Resident Assessment and Care Screening (MDS) is the core functional assessment instrument in the RAI and covers such domains as physical functioning in the activities of daily living (ADLs), cognition, continence, mood, behaviors, nutritional status, vision and communication, activities, and psychosocial wellbeing. The purpose of the MDS assessment is to identify a resident's strengths, preferences, and needs in key areas, and provide a holistic and comprehensive picture of the resident's functional status. ${ }^{5,6}$ In addition, the RAI includes 18 problem-focused Resident Assessment Protocols (RAPs) that specify additional, highly focused assessment to identify treatable causes of conditions that are common among nursing home residents or that represent a severe health risk. RAP assessment is required if a resident's completed MDS suggests a problem, risk for decline, or potential for improved function. The RAI system also includes a form on which staff summarizes decisions about care planning and notes the location of any planned intervention, a training manual, ${ }^{7}$ and mandated quarterly review forms. Thus, it is a system that in content and focus goes well beyond what most facilities were doing pre-OBRA. ${ }^{8,9}$ The RAI and other OBRA requirements went into effect as part of federal law in October 1990, although delays meant most states required facilities to implement the RAI between the spring and fall of 1991.

Before passage of OBRA, studies found widespread deficiencies in process quality in the nation's nursing homes. Process quality encompasses interactions between staff and residents and the appropriateness of care and services provided to residents, including assessment, care planning, and care provision. ${ }^{10-13}$ In long-term care facilities, where residents receive care for months or years, the process of care is especially relevant, affecting residents' health, functional status, and the quality of their daily lives. ${ }^{13,14}$ Studies found that pre-OBRA, assessment information was often inaccurate, incomplete, and unrelated to the care plan. ${ }^{2,5,8}$ For example, mood problems and delirium were underdiagnosed and undertreated among nursing home residents. ${ }^{15,16}$ Poor care practices were all too common and included use of physical restraints, inappropriate use of psychotropic medications, overuse of urinary catheters, deficient treatment of incontinence, inadequate prevention and resolution of pressure ulcers, inattention to nutritional problems, a lack of regard for hearing, vision, and dental problems, and inadequate psycho- social interventions, including behavior management programs. ${ }^{17-30}$ The RAI, with comprehensive assessment and a focus on identifying treatable, reversible causes of functional limitations and on restoring and maintaining function, was designed to effect changes in these practices. This research investigates whether changes in process quality occurred.

\section{METHODS}

The evaluation of the RAI employed a quasi-experimental design, using a multistage probability-based sample of facilities and residents, to examine the effect of RAI implementation on various indicators of process quality.

Data

\section{Sampling}

Data were collected on the implementation of the RAI through site visits in 254 facilities located in 42 counties in or adjacent to 10 metropolitan statistical areas (MSAs), each in a different state. The 10 states were chosen so that the RAI would be evaluated in environments that differed in Medicaid reimbursement strategy, rate level, and the statewide average nurse staffing level, factors that might influence the ability of facilities to fully implement the RAI. The MSAs included: Oakland, CA; Hartford, CT; Des Moines, IA; Baltimore, $\mathrm{MD}$; Minneapolis, MN; Cleveland, $\mathrm{OH}$; Portland, OR; Nashville, TN; Dallas, TX; and Virginia Beach, VA.

The evaluation included four waves of data collection (two pre- and two postimplementation) by research nurses in each of the participating facilities. Waves 1 (fall, 1990) and 3 (spring, 1993) provide the data for the analyses included in this examination of process quality. We retained $95 \%$ of the 267 facilities that participated in Wave 1 through all four data collection rounds, and only data on residents from 254 facilities that participated in all waves were used in the present analysis. Data were gathered on 2170 randomly selected residents in the pre-RAI cohort and 2088 in the post-RAI cohort. Resident-level results are based on weighted data. The study sample was a multistage sample, with residents clustered in facilities. Standard statistical software that assumes simple random sampling provides biased variance estimates when used with such data. Thus, we used SUDAAN, a special statistical software that generates appropriate variance estimates for data from complex samples. ${ }^{31}$ In addition, the variances for the resident-level results are estimated using the finite population correction because the sample included nearly all facilities in the smaller MSAs, as discussed in greater detail elsewhere. ${ }^{32,33}$

\section{Data Collection}

The evaluation team hired and trained registered nurses (RNs) with experience in geriatric nursing to collect the resident-level data, using an MDS-based tool. The research RNs received 4 days of training on the data collection protocols. This included instruction in (1) how to conduct an MDS assessment, using the items, definitions, response categories, data sources, and procedures specified in detail in the RAI training manual used by facilities ${ }^{7}$; $(2)$ training on how to review and evaluate care planning processes and individual resident care plans; (3) one-half day of practice assessments in nursing facilities; and (4) debriefing on those assessments, as well as consent procedures and similar related topics, such as selecting the resident sample. As specified in the RAI training manual, the MDS is not a set of questions. Rather it is a set of 
items with specified definitions and response categories, as well as a systematic process for arriving at a response category for a given item. Except for the measure of "comprehensiveness of care plans," all of the process quality measures described below are items from the MDS. Thus, the research RNs followed the protocols specified in the RAI training manual for scoring each item. ${ }^{7}$

Detail on each MDS item can be found in the RAI training manual in the section on item-by-item specifications. ${ }^{7}$ However, in general, the process involves collecting information from multiple sources. For most items, except as noted below, this process involved interviews with and observation of each sampled resident and interviews with several staff caregivers for each sampled resident (e.g., nursing assistants on multiple shifts who provided direct care to the resident, therapists, unit charge nurse, activities staff), including collection of information from staff across all shifts. It also included review of relevant medical records, such as assessments (comprehensive and discipline-specific), physicians' orders, treatment and care plans, nursing progress notes, and medication records. If a resident was unable to communicate, the research nurse only observed the resident. The observations centered around the MDS items selected (e.g., behaviors, mood signs, and the amount of assistance the resident was receiving with activities) using the indicators and protocols specified in the RAI manual. However, because the MDS assessment protocols specify that items cover what happens across all shifts ( 24 hours a day) over several days (e.g., typically 7), this observation yielded only one of several pieces of information, with additional information from staff caregivers and records. When these multiple sources disagreed, the research nurses were instructed to seek additional information so that they could reach an informed conclusion and score the item. This is an established and systematic process using well-defined items, and one which numerous trials have found to produce reliable data. ${ }^{6,34,35}$ Most of the selected process quality measures achieved kappas or intraclass correlations greater than 0.6 and all exceeded $0.4 .^{6}$ In each wave of data collection, research RNs spent about 4 days in each facility collecting data on an average of eight residents.

\section{Measures}

\section{Dependent Variables}

The analysis focused on several indicators of process quality. The prevalence of all measures in the 1990 and the 1993 cohorts are the dependent variables in the analysis, with no attempt to aggregate these into a single measure.

Accuracy of Information. One of the key goals of OBRA in mandating the RAI was to improve the quality of information available in residents' medical records upon which critical care decisions are based. Thus, we examined changes in the accuracy (and comprehensiveness) of information on the residents' functional status. As part of the pre- and post-RAI implementation data collection, research RNs used only the available information in residents' records to score them on 23 functional status items, including seven ADLs; urinary continence; cognitive status (short-term memory and decision-making); behaviors (wandering and disruptive behaviors); verbal expression of sad mood; communication (understands others); falls in the last 30 days; presence of a pressure ulcer; contractures; hand dexterity; functional vision; use of trunk restraints; and whether the resident was awake during most of the day shift. The records reviewed included each resident's comprehensive and discipline-specific assessments, physician orders and notes, nursing progress notes for the last 3 months, medication records, and the resident's care plan. The residents' scores on the 23 items, based on the information in these records, were compared to the information the research RNs subsequently collected by performing a full functional assessment to score the resident on all MDS items. This full MDS assessment was based on information the research RNs generated from multiple sources, as described above (i.e., using data from records, interviews with staff across multiple disciplines and shifts, and interviews with and observations of the resident). We performed two types of analysis on these data, first examining only changes in accuracy for those items for which information was available in the facilities' records. Then, we examined changes in accuracy with missing items in the records treated as inaccurate, as were scores that did not agree with those generated by the full MDS assessment. Because the results were the same, we present only the findings for the latter construct because we believe it is appropriate to treat missing data on key areas of resident functioning (e.g., mood) as "inaccurate," particularly because such items are necessary to implement and monitor the effects of individualized, restorative care plans.

Comprehensiveness of Care Plans. The research RNs reviewed each sampled resident's comprehensive and discipline-specific care plans, as well as other documents the facility staff identified as including care plan information. This was done to determine the extent to which residents' problems, their risk for decline or problem development, and their potential for improvement were being addressed in the 18 areas displayed in Table 2 . The protocol allowed for multiple problems to be addressed by a single care plan intervention (e.g., mood and behavior problems that facility staff thought were related might be addressed by a single intervention), if the short or long-term goals for that intervention indicated that such multiple conditions were being addressed. Thus, we compared the proportion of residents for whom these conditions were addressed in care plans preand post-RAI implementation. For this process quality measure, we relied only on written documentation to determine whether care plans were more comprehensive.

Use of Physical Restraints. Use of trunk or limb restraints or being confined to a "chair that prevents rising" (e.g., geriatric chair with fixed lap-board) during the preceding 7 days was defined as restraint use. Use was determined from review of the records, interviews with staff across all shifts who provided direct care to the sampled residents, asking whether such devices were in place at any time during the last 7 days, and interviews with and episodic nonscheduled observation of the residents over the course of 4 days. Most restraint use occurs on a daily basis; moreover, these items have high reliability $(P>.7) .^{6,17,36}$

Psychotropic Drug Use. Information was collected from medication records on the residents' use of three different classes of psychotropic drugs during the preceding 7 days: antipsychotics, antianxiety/hypnotic agents, and antidepressants. We examined these as separate measures in view of the different indications for each and the different implications for care quality; however, we report the findings in only two categories, grouping receipt of antipsychotics and antianxiety/hypnotics together for purposes of presentation. 
Treatment of Sad Mood. We examined the proportion of residents who were not receiving antidepressants or therapy/ services from a mental health professional but had a diagnosis of depression or observable signs and symptoms of persistent sad or anxious mood over the last 30 days and either decline or no improvement in mood over the last 90 days. Because depression in typically underdiagnosed among nursing home residents ${ }^{15}$ and because many residents have cognitive impairment and cannot respond to a questionnaire, the research RNs used MDS items and asked nursing assistants who provided hands-on care to the sampled residents, nurses, activity staff, and dietary staff whether the resident presented any relevant signs and symptoms over the last 30 days (e.g., verbal expressions, such as feelings of worthlessness, that life is not worth living; persistent anger with self or others; repetitive anxious complaints; repetitive, anxious behaviors, such as pacing, hand wringing; recurrent crying or tearfulness; withdrawal from activities, self-care, family, friends; change in sleep patterns). They also spoke with residents who could communicate, discussing topics such as their relationships with staff, other residents, and family, their involvement in the life of the facility, their preferences for activities, their customary routines, and so on, as well as more direct probes about how they were feeling. Receipt of therapy and of medications was based on medication sheets and information in the residents' medical records (e.g., physician notes).

Behavior Management. We examined the proportion of residents with behavioral symptoms (i.e., wandering, physical or verbal aggression, resisting nursing care, and socially inappropriate behaviors, such as yelling, disrobing, smearing feces) who were receiving a structured behavioral intervention that was not limited to use of physical restraints or psychotropic medications. The RNs used records and interviews with direct staff caregivers to determine whether any behavior management program was in place. Interventions to prevent behaviors as well as to manage manifestations were counted, as long as they were (1) related to known causes of an individual resident's behavioral manifestations (e.g., "when Mrs. S resists bathing, we dol.I.I.," (2) used systematically throughout the preceding week, (3) known to direct staff caregivers for the resident across multiple shifts (not merely written in the care plan), and (4) included more than reliance on only restraints or psychotropic medications.

For the next five measures, the research RNs used the standard MDS protocols described above. Specific detail on each item (i.e., the item, definition, response categories, coding instructions, and specified sources of information, as well as suggested probes for interviews and observation) may be found in the RAI training manual. ${ }^{7}$

Protective Or Preventive Skin Care. Because so many residents were at risk for the development of pressure or stasis ulcers or had friable skin, we examined the proportion of residents receiving protective or preventive skin care.

Attention To Sensory Deficits. We examined the proportion of residents with hearing problems for whom a hearing aid was present and used during the seven days preceding the data collection. Similarly, we examined the proportion of residents with vision problems who were not blind and who had a visual appliance.

Attention to Psychosocial Well-Being. Attention to psychosocial well-being encompasses a variety of processes, such as the residents' involvement in decision-making, relationships with family, staff, and other residents, and so on. It also includes participation in activities. For this analysis, we examined the proportion of residents who were awake for significant periods of the day who had little or no activity involvement. Activities were defined to include large and small group activities, as well as individual activities, such as reading. Nursing assistants, activities staff, and residents were sources of information and observation for these items, and time spent in ADL activities, therapy and meals was excluded from the calculation of available time for activities.

Treatment $O f$ Bowel and Bladder Incontinence. Unfortunately, the data we collected on services were too limited to reveal the full scope of the facilities' adherence to the RAP assessment processes. For example, the only reliable indicator of testing for urinary tract infections and fecal impaction covered testing only in the last 14 days and was considered too limited an indicator of change in process quality. Thus, we relied on examining whether there was any change in management of incontinence, comparing the use of bowel and bladder retraining programs and scheduled toileting in the two cohorts. Prevalence was determined based on information from multiple sources, particularly interviews with aides who regularly assisted residents with toileting.

Use of Indwelling Urinary Catheter. We examined the proportion of residents for whom an indwelling urinary catheter was used during the 7 days preceding the data collection, based on review of physician treatment orders, nursing progress notes, interviews with direct care staff, and observation of the resident (e.g., presence of tubing).

For the remaining two items, the data collection protocols specify that there must be supporting documentation in the residents' records.

Advance Directives. We examined the proportion of residents who had supporting documentation in their medical record in compliance with state laws specifying any type of advance directive (e.g., living will, do not resuscitate, do not hospitalize, feeding or medication restrictions). We did not rely on nursing notes or summary charts at the resident's bedside.

Use of Therapies. We used two measures, receipt of any therapies during the preceding 7 days (i.e., speech, occupational, physical, or psychological); and an average number of days the resident received any of these therapies during the preceding 7 days. As expected, both behaved similarly, and we report the analysis for only the second measure.

\section{Independent Variables}

The principal independent variable is cohort; it serves as a proxy for the implementation of the RAI and other salient OBRA provisions. Thus, in the logistic models, the primary independent variable was an indicator of "post-RAI cohort," testing the hypothesis that there were positive changes in process quality after implementation of the RAI. We also introduced other variables as covariates in the multivariate analyses to control for any changes in resident characteristics that might affect the process of care residents received. These included resident age, gender, length of stay in the nursing home, and score on a summary scale of dependence in ADLs. This scale combines the resident's scores on self-performance in transfer, locomotion, eating, toileting, dressing, and bathing, with their score on urinary continence. Residents with the lowest score would be continent and independent in the other six ADLs, whereas the highest score would be for a resident who was totally dependent in eating and at least two 
other ADLs or totally dependent in one ADL and totally incontinent. We also introduced a covariate representing the resident's score on the Cognitive Performance Scale (CPS) ${ }^{\oplus}$ which has been shown to replicate scores on the Folstein Mini-Mental Status Exam and the Test for Severe Impairment and scores a resident into one of seven categorical variables ranging from "intact" to "very severely impaired." ${ }^{37}$ We also used a modified version of an index of case-mix intensity using a modified Resource Utilization Group (RUG-III) classification. ${ }^{38}$ The case-mix intensity variable reflects variance in weighted nursing care time, or the relative "costliness" in staff time of residents in each of the RUG-III groups. Because these global measures of function may not adequately adjust for the medical or behavioral factors that predispose staff to physically restrain residents or physicians to prescribe psychotropic drugs for residents, several other MDS items were used as covariates, including indications of Alzheimer's disease or other dementias, a prior history of psychiatric problems, a diagnosis of Parkinson's disease, a prior stroke, balance problems, and a diagnosis of depression. Finally, we included as covariates variables representing a set of external factors that might be expected to affect process quality in nursing homes, including facility size and ownership, location in an MSA, and the state.

\section{Analytic Approach}

The cohorts were first compared using bivariate analyses with respect to the major independent variable (i.e., RAI and OBRA implementation) and the dependent variables. For each measure of process quality, we constructed a contingency table and examined the relationship between cohort and the dependent variable using the chi-square statistic. For the residents receiving psychotropic medications, the characteristics of residents from each cohort who were receiving each type of intervention were compared, using stratified analyses. Finally, the effect of cohort on the prevalence of each process quality indicator was tested using multivariate logistic regression and controlling for selected covariates. The research hypothesis under consideration for each dependent variable is that process quality improved in the post-RAI period. Because one might reasonably conclude that we were doing repeated tests of the same hypothesis, albeit with different measures of process quality each time, we used a Bonferroni adjustment and thus adopted more conservative alphas than 0.05 in testing for significance of any change in comprehensiveness of care plans in the 18 areas selected (i.e., $P<.0028)$, as well as in testing the significance of differences in the 14 other process quality indicators (i.e., $P<.0036$ ).

\section{RESULTS}

\section{Accuracy of Information on Residents' Status and Comprehensiveness of Care Plans}

Table 1 presents the results of a comparison of the accuracy of information in the facility's records in the preand post-RAI implementation periods. As shown, the level of accuracy increased significantly. In the pre-RAI period, only $18 \%$ of residents had accurate information in their records for all or almost all of the 2.3 items (i.e., for $90-100 \%$ of the items). In the post-RAI period, the proportion of residents with accurate information on all or almost all of the items was almost three times higher $(49 \%)$.

We also found that changes in accuracy were associated with some covariates. For example, information in residents'
Table 1. Summary Measures for the Percent of Accurate Information Available on Residents in Facility Medical Records

\begin{tabular}{lccc}
\hline & $\begin{array}{c}\text { Percent } \\
\text { of Items } \\
\text { Assessment Area* }\end{array}$ & \multicolumn{2}{c}{$\begin{array}{c}\text { Percent of } \\
\text { Residents }\end{array}$} \\
\cline { 4 - 5 } Accurate & 1990 & 1993 \\
\hline All items (23) & $0-59$ & 30.6 & 8.0 \\
& $60-89$ & 51.8 & 43.5 \\
Functional/continence (12) & $90-100$ & 17.6 & 48.6 \\
& $0-59$ & 36.4 & 15.9 \\
& $60-89$ & 37.9 & 28.2 \\
Cognitive/psychosocial (5) & $90-100$ & 25.7 & 55.9 \\
& $0-60$ & 41.4 & 14.3 \\
& 80 & 29.2 & 26.1 \\
& 100 & 29.4 & 59.6 \\
\hline
\end{tabular}

* The probabilities of the chi-squares for the three contingency tables formed by the data presented above (i.e., accuracy of all items by time, accuracy of functional/ continence items by time, etc.) were less than .0001 .

records in States having Medicaid case mix payment systems was more accurate in both 1990 and 1993. However, this did not change the significant impact of cohort on improvements in accuracy; the records in these States also had statistically significant improvements in accuracy after the implementation of the RAI. Indeed, the overall effect of RAI implementation was to reduce the amount of variation in accuracy rates among the states.

Table 2 presents information on the degree to which problems identified by the 18 RAPs were addressed in resi-

Table 2. Percent of Residents for Whom a Problem or Risk Factor Is Addressed in Their Care Plan

\begin{tabular}{|c|c|c|c|}
\hline \multirow[b]{2}{*}{ RAP Area } & \multicolumn{2}{|c|}{$\begin{array}{l}\text { Percent with } \\
\text { Problem } \\
\text { Addressed }\end{array}$} & \multirow[b]{2}{*}{ Significance } \\
\hline & $\begin{array}{c}1990 \\
\text { Cohort }\end{array}$ & $\begin{array}{c}1993 \\
\text { Cohort }\end{array}$ & \\
\hline Delirium & 36.2 & 29.1 & $>.051$ \\
\hline Cognitive loss & 73.7 & 86.5 & $<.001$ \\
\hline Visual function & 46.4 & 61.3 & $<.001$ \\
\hline Communication & 60.9 & 72.3 & $<.001$ \\
\hline ADL rehabilitation & 79.5 & 92.3 & $<.001$ \\
\hline Incontinence/catheter & 75.2 & 84.3 & $<.001$ \\
\hline Psychosocial Wellbeing & 74.9 & 79.8 & $>.146$ \\
\hline Mood state & 62.1 & 72.6 & $<.001$ \\
\hline Behavior & 73.4 & 86.2 & $<.001$ \\
\hline Activities & 78.1 & 84.6 & $>.012$ \\
\hline Falls & 54.9 & 72.2 & $<.001$ \\
\hline Nutritional status & 88.0 & 92.3 & $<.001$ \\
\hline Feeding tubes & 82.5 & 93.9 & $>.005$ \\
\hline Dehydration & 53.3 & 65.5 & $<.001$ \\
\hline Dental care & 30.3 & 47.3 & $<.001$ \\
\hline Pressure ulcers & 65.5 & 48.7 & $<.001$ \\
\hline Psychotropic drug use & 43.2 & 75.7 & $<.001$ \\
\hline Physical restraints & 68.4 & 74.9 & $>.054$ \\
\hline
\end{tabular}

The significance level, with correction for multiple comparisons, was $P<.0028$. The significance levels are based on results of multivariate logistic regression. 
dents' care plans. In twelve of the 18 RAP areas, residents in the post-RAI period were significantly more likely to have potential problems addressed in their care plans. In three areas (psychosocial well-being, activities, and physical restraints), there was an increase in the proportion of residents with a care plan intervention, but the differences were not statistically significant whether one uses a traditional alpha level (i.e., $P<.05$ ) or a more conservative level of significance (i.e., $P<.0028$ ). The increase in addressing problems or risks associated with Feeding Tubes is significant using an alpha of 0.05 but not statistically significant using the more conservative significance level. In two areas, Delirium and Pressure Ulcers, fewer residents have care plan interventions in the post-RAI period; however, only the reduction in care planning for pressure ulcers was statistically significant using the more conservative level of significance.

\section{Effects on Other Indicators of Process Quality}

On 14 other indicators of process quality, differences between the pre- and post-RAI cohorts provided some evidence of better quality of care in the post-RAI period. However, on most indicators, there was no evidence of change. Table 3 presents the results when we compared selected indicators of the process of care from the two periods. First, this table presents the prevalence of various care processes in the two cohorts. Although only the unadjusted bivariate prevalences are displayed for the two time periods, the statistical significance of the differences between these prevalences, shown in the last column, was based on logistic regression models.

For five of the 14 measures, there was a significant change in the direction of better process quality. This includes reductions in poor care practices, such as use of physical restraints (a $25 \%$ decline) and use of indwelling urinary catheters ( $29 \%$ reduction). It also includes increases in appropriate care practices, such as activity involvement among residents $(28 \%$ reduction in prevalence of residents with no activity involvement), presence of advance directives $(64 \%$ increase), and use of toileting programs for residents with bowel incontinence ( $37 \%$ decrease in the proportion of such residents with no program). In two other areas, use of behavior management programs and presence and use of hearing aids, the improvements were significant at the .05 level but not with the more conservative alpha level.

In seven other areas of process quality, no significant change was observed. These included use of psychotropics (two measures); preventive skin care; treatment of depression or sad mood; use of a toileting program for residents with urinary incontinence; provision of glasses (or lens) to residents with inadequate vision; and provision of therapies, in which a decline occurred that was significant at the .05 level but not at the more conservative level (i.e., $P>.0036$ ).

Our focus in these analyses has been on examining the relationship between the introduction of the RAI and other OBRA provisions and changes in process quality, rather than on examining the association between other factors and differences in process quality. Thus, we do not report much detail on the effects of the covariates introduced in our model, because our primary interest is in whether they rather than cohort explain differences in process quality. However, reducing inappropriate use of psychotropics and physical restraints was a key element of the OBRA provisions. Thus, we performed additional analyses on these measures. (For presentation simplicity, these results are discussed but not displayed; however, the analyses are available from the authors.)

As can be seen in Table 3, there was no significant change in the use of two general categories of psychotropic medica-

Table 3. Differences in the Prevalence of Process Quality Indicators

\begin{tabular}{ll} 
Process Quality Measure & $\begin{array}{c}\text { Prevalence (\%) } \\
19901993 \\
\text { Cohort Cohort Significance }\end{array}$ \\
\hline
\end{tabular}

Process Quality Indicators, Using Total Resident Population

Any advance directives

Indwelling urinary catheter

Not involved in activities

Protective/preventive skin care

Physical restraints used

Use of antipsychotics or antianxiety/hypnotics

Use of antidepressants

Average number of days of therapy

Process Quality Indicators, Using Relevant Subsets of the Total Population

Residents with inadequate hearing and no hearing aid or hearing aid not used

Resident with inadequate (but some) vision without glasses or contact lens

Residents with no ostomy and bowel incontinence but no toileting program

Residents with urinary incontinence and no indwelling catheter but no toileting program

Residents with persistent sad mood or depression diagnosis and no mood improvement

in the last 90 days who are not getting antidepressants or psychological therapy

Residents with a behavioral symptom who do not receive a behavior management program

$\begin{array}{rrr}35.2 & 57.7 & <.001 \\ 9.8 & 7.0 & <.001 \\ 16.6 & 12.0 & <.001 \\ 56.7 & 60.9 & >.009 \\ 38.0 & 28.5 & <.001 \\ 27.1 & 29.3 & >.163 \\ 12.6 & 14.1 & >.221 \\ 0.17 & 0.12 & >.016 \\ & & \\ 79.8 & 70.9 & >.018 \\ 22.2 & 26.4 & >.051 \\ 13.9 & 8.8 & <.001 \\ 58.7 & 57.6 & >.749 \\ 65.5 & 57.5 & >.080 \\ & & \\ 22.0 & 16.1 & >.011 \\ & & \end{array}$

The significance level, with correction for multiple comparisons, was $P<.0036$. The significance levels are based on results of multivariate logistic regression. 
tions, antipsychotics and antianxiety/hypnotics. In analyses not shown here, we analyzed the two classes separately and found a slight increase in the rate of antipsychotics and no change in the use of hypnotics. However, using multivariate logistic regression, we controlled for changes in resident characteristics between the two cohorts, including the prevalence of anxiety disorders, depression, and dementias, and found that the differences were not statistically significant. There was also a slight increase in the use of antidepressants, but again, the multivariate analysis demonstrated that the change was not statistically significant. As expected, scores on the ADL index and CPS ${ }^{(}$, the diagnostic indicators, and age were significantly related to use of antipsychotic medications. Similar results were obtained for use of hypnotics, with age and ADI. independence inversely related to use of hypnotics. Otherwise, only having an indication of anxiety noted in the chart was significantly related to increased hypnotic use. For anti-depressants, residents with CPS scores indicating greater levels of cognitive impairment, residents who were younger, and those who had been in the facility longer had higher use. Not surprisingly, the biggest predictor of antidepressant use was diagnostic evidence of depression.

In the logistic models examining changes in the use of physical restraints, we found that residents who scored as more impaired on the CPS and ADL scales and who had a diagnosis of Alzheimer's, other dementias, or Parkinson's disease were more likely to be restrained in both cohorts. However, the rate of restraint use observed in the 1993 cohort was somewhat more concentrated in the more impaired ADL and CPS levels than in 1990. Thus, residents who were the least impaired ADL and CPS levels experienced proportionally greater reductions in restraints than did more functionally impaired residents. Thus, for example, residents with an intact score on the CPS went from $9.1 \%$ restraint use to $4.0 \%$ - a $63 \%$ reduction; however, use of restraints among residents scoring as severely impaired on the CPS level changed from 63.9 to $56.2 \%$, a decline of only $12 \%$. (Greater detail on the predictors of restraint use pre-OBRA may be found elsewhere, ${ }^{36}$ as can data showing a continued decline in restraint use through 1994 to an estimated rate nationally of $20 \% .{ }^{39}$ ) However, none of these covariate effects overwhelmed the effect of cohort on reduced use of restraints.

\section{DISCUSSION}

The changes in accuracy of information and comprehensiveness of care planning are encouraging. On the other hand, there is still room for improvement. As shown in Table 1, only about half the residents have accurate information in their records for all or almost all of the 23 key items reflecting residents' functional status, but the need for clinically relevant and accurate information to use in care planning and monitoring means that the records of all residents should approach these levels of accuracy for all items. Similarly, although the overall positive effect of the RAI on comprehensiveness of care plans is encouraging, there are three limitations to this finding. First, the improvement is not uniform. The areas in which no improvement was found, such as delirium and pressure ulcers, warrant further investigation. The fact that, during the evaluation period, there was no increase in prevalence of pressure ulcers or mortality and were reductions in hospitalizations, as reported elsewhere, suggests facilities may be responding appropriately. ${ }^{40,41}$ For example, increased attention to nutrition and hydration, as shown in Table 2 , and reductions in restraint use may be adequately addressing risk factors for pressure ulcers. However, more study is needed on care of these critical conditions in nursing homes. Second, as with accuracy, there seems to be room for improvement in care planning across all 18 areas. This is especially true because the study protocol for examining comprehensiveness of care plans addressed only what was found in records. Investigation is needed to determine what was actually implemented and whether it met standards for clinically appropriate care and conformed with residents' preferences.

The fact that we found significant improvements in process quality for only five of 14 measures also warrants further investigation. Some are easily explained. For example, the available evidence from other studies and data sets suggests that there was a substantial reduction in psychotropic drug use after the passage of OBRA in 1987. Pre-OBRA prevalence rates ranged from a low of 33 to a high of $74 \%$, with more common estimates in the 50 to $60 \%$ range, whereas our data and others show post-OBRA rates that are substantially lower. ${ }^{19,21,42,43}$ However, the change seems to have occurred in the interim between passage of OBRA and its implementation in 1990 to $1991 .^{18}$ Similarly, Medicare and Medicaid coverage and eligibility policies may explain the lack of effect on use of hearing and visual appliances and therapies, although such potential explanations are hardly comforting in terms of their impact on the functional well-being of nursing home residents. However, identifying the factors that contribute to lack of improved process quality and examining their impact remains a critical task. The failure to increase appropriate therapies for nursing home residents with depression or sad or anxious mood problems continues to be a vexing problem that warrants especially serious investigation, given the consequences in morbidity and mortality of untreated depression in older people.

In terms of no significant change in scheduled toileting for residents with urinary incontinence, two explanations are possible. First, facilities may be appropriately pursuing more aggressive interventions to restore a higher level of continence, and we simply selected a poor measure of process quality. Alternatively, facilities may still be failing to provide an appropriate level of services to help keep residents dry. The fact that there was a significant reduction in resident decline in the area of urinary incontinence, as reported elsewhere, ${ }^{3.3}$ lends credence to the first explanation. However, the under-provision of toileting programs, including scheduled toileting and bladder retraining, has been observed and reported for years in nursing homes. ${ }^{2.3-26}$ Thus, this is a critical area that warrants further investigation aimed at clarifying both treatment and management of incontinence.

\section{Limitations of the Findings and Attribution of Change to the RAI}

There are two potential limitations to these findings. First, as we noted earlier, our methodology for assessing the effect of the RAI on comprehensiveness of care plans relied on review of written care plans and did not entail a determination of other important questions about the quality and implementation of the care plans. Thus, we have only one piece of the puzzle when considering effects on care planning. Second, our measures do not represent a full range of the processes of care that may be important to the quality of care received by residents and may affect their outcomes. More- 
over, they are insufficient to capture adequate indicators of process quality in the area of quality of life. For example, we do not measure such critical aspects of process quality as the nature of staff-resident interactions nor resident satisfaction. Given the goals of the OBRA nursing home reforms, these aspects of process quality and whether they have improved deserve further study.

Many of the improvements, particularly in accuracy of information in records and in comprehensiveness of care plans, seem to be clearly attributable to the implementation of the RAI, independently of other OBRA provisions or any general trend lines. First, the structure of the RAI explicitly addressed the deficits that existed before in these areas and included both mandates and clinically relevant educational materials intended to show facilities how to achieve improvements in these areas. Second, these empirical findings of improvements and their attribution to the RAI are supported by the reports of facility nursing staff and administrators who have been surveyed about the effects of the RAI on quality, as well as by State and Federal surveyors, ombudsmen, and resident family members. ${ }^{4,45,46}$ For example, significant majorities of administrators and directors of nursing who were surveyed both before and after implementation of the RAI reported that the MDS, RAPs, and training manual had improved the quality of assessment, problem identification, and care planning in their facilities. ${ }^{44}$

We also examined the potential effect of other factors that might be expected to account for the observed improvements in process quality, such as facility characteristics and State effects in the multivariate models. Although some had an independent effect, they did not change the significant effect of cohort. In addition, we considered whether an increased physician role might have altered information accuracy and care plan comprehensiveness. However, the percent of facilities in which physicians (including the medical director) participated in developing the plan of care or attending care plan meetings increased from only $2 \%$ in 1990 to $4 \%$ in 1993. This suggests that changing physician roles in nursing homes would not account for the magnitude of the observed changes. ${ }^{47}$ As noted in the discussion on use of psychotropics and restraints, we also looked for trend lines; however, even with psychotropics, in which the change took place before OBRA and RAI implementation, the change seems to have begun with the passage of OBRA, not earlier. For other aspects of process quality examined, the transformations all seem to be post-OBRA and RAI implementation. Increased use of advance directives is one possible exception, because it may have also been affected by the 1990 passage of the "Patient Self-Determination Act"; however, there is no apparent way to disentangle this from RAI effects. ${ }^{48}$

Finally, it is important to note that the effects seen are not an artifact of the standardized MDS instrument being available in the post-RAI period and affecting the research RNs' judgments about the accuracy of information on resident strengths, preferences and care needs. Certainly, the presence of the MDS did affect accuracy by decreasing the amount of missing information on key functional areas, such as those covered in mood, behaviors, and cognition, which were not addressed by many facilities in the standardized forms they used pre-RAI. Yet even in areas such as ADL performance and continence that were part of pre-RAI assessment information routinely assessed and recorded by facilities in residents' records, there were significant post-RAI improvements in the accuracy and completeness of information. Further, the research RNs, most of whom participated in all waves of data collection, were trained for 4 days on appropriate protocols for conducting the data collection and explicitly informed about the danger of assuming that information recorded on an MDS assessment form was accurate. In both waves of data collection, they followed the same protocols. Moreover, if the research RNs had simply assumed that the post-RAI data were accurate because they were found on the MDS, one would not expect to find the variation that still exists in accuracy across items and domains, as shown in Table 1. Instead, all items would have achieved higher levels of agreement and a more uniform level of accuracy across items.

Attributing observed changes in other aspects of process quality to the RAI is more problematic. Randomized trials are not possible when change is the result of federal legislation, and it is impossible with our study design to determine the precise effect of the RAI compared to the effects of other OBRA provisions and the increased focus of the survey process on care pratices, such as restraint reduction, and on resident outcomes.

\section{CONCLUSIONS}

Many prior studies have found that comprehensive geriatric assessment has positive effects on the health and wellbeing of older persons. ${ }^{49-52}$ The RAI has extended that practice to nursing homes in a more systematic and widespread way than ever before, with similar positive results. In the area of improvements in process quality, the case for the positive effect of the RAI's implementation is compelling. The MDS and RAPs specify clear assessment and care planning processes that should logically lead to the results we observed in accuracy of information on residents' functional status and in increased comprehensiveness of care plans. The RAl's RAPs both identify problems, risk factors, and potential for improved function and also specify further assessment and identification of treatable causes for the conditions. Thus, the logic of the RAI structure suggests that there is a strong nexus between the introduction of the RAI and the observed improvements in process quality.

The OBRA reforms and introduction of the RAI constituted an unprecedented implementation of comprehensive geriatric assessment in Medicare- and Medicaid-certified nursing homes. The evaluation of the effects of these interventions demonstrates significant improvements in the quality of care provided to residents. At the same time, these findings suggest that more needs to be done to improve process quality and resident outcomes. The RAI has been shown to be one tool that facility staff, therapists, pharmacy consultants, and physicians can use to support their continuing efforts to provide high quality of care and life to the nation's nursing home residents.

\section{REFERENCES}

1. Smith v. O'Halloran, 557 F Supp. 289 (D Colo. 1983), rev'd sub nom Smith versus Heckler, 747 F.2d 583 (10th Cir. 1984).

2. Institute of Medicine Improving the Quality of Care in Nursing Homes Washington, DC. National Academy of Sciences Press, 1986.

3. Hawes $C$. The Institute of Medicine study: Improving the quality of nursing home care, In: Katz PR, Kane RL, Mezey MD, eds. Advances in Long-Term Care. New York: Springer, 1991, pp 147-168.

4. Contract DHHS 500-88-0055 was with the Health Standards and Quality Bureau of the Health Care Financing Administration. The project team was led by Research Triangle Institute in North Carolina, with its subcontractors, the Social Gerontological Research Center, Hebrew Rehabilitation Cen- 
ter for Aged, in Boston, MA; the Center for Gerontology and Health Care Rescarch, Brown University, Providence, RI, and the Institute of Gerontology, University of Michigan, Ann Arbor, MI.

5. Morris JN, Hawes C, Frics BE et al. Designing the national resident assessment instrument for nursing homes. Gerontologist 1990;30:293-307.

6. Hawes C, Morris JN, Phillips C ct al. Reliability estimates for the Minimum Data Set for nursing home resident assessment and care screening (MDS). Gerontolegist 199.5;35:172-178.

7. Morris JN, Hawes C, Murphy K et al. Resident Assessment Instrument Training Manual and Resource Ciuide. Natick, MA: Fliot Press, 1991.

8. Hawes C, Phillips CD, Morris JN, Mor V, Fries BF. Reliability and Validity of the Nursing Home Resident Assessment Instrument (RAI): Report on the Field Testing of the RAI. Research Triangle Park, NC: Research Triangle Institute, 1991.

9. Mor V, Laliberte I, Drugovich M. Review of Standardized Assessments Used in U.S. Nursing Homes. Providence, RI: Center for Gerontology and Health Care Research, Brown University, 1989.

10. Donabedian A. Evaluating the quality of medical care. Milbank Mem Fund Q 1966:4:166-206.

11. Donabedian A. Quality asscessment and assurance: Unity of purpose, diversity of means. Inquiry 1988:25:6-12.

12. Wyszewianski L. Quality of carc: Past achievements and future challenges. Inquiry 1988;25:13-22.

13. Kane RA, Kane RI.. Long-term care: Variations on a quality assurance theme. Inquiry 1988;25:1.32-146.

14. Sloane PC., Pickard C(i. Custodial nursing home care: Sctting realistic goals, J Am Geriatr Soc 1985;33:864-868.

15. Rovner BW, Cierman PS, Brant 1.J et al. Depression and mortality in nursing homes. JAMA 1991;265:993-996.

16. Liston E.H. Delirium in the aged. P'sychiatr (lin North Am 1982;5:49-66.

17. Fvans LK, Strumpf NE. Tying down the elderly: A review of the litcrature on physical restraints. J Am Gicriatr Soc 1989;37:65-74.

18. Kane RL, Williams CC, Williams TF et al. Restraining restraints: Changes in a standard of care. Annu Rev Public Health 1993;14:545-584

19. Ray WA, Federspiel (F, Schaffner W. A study of anti-psychotic drug use in nursing homes: Epidemiological evidence suggesting misuse. Am J Public Health 1980;70:48.5-491.

20. Howard J. Medication procedure in a nursing home: Abusc of PRN orders. I Am Geriatr Soc 1977;25:8.3-84

21. Harrington C., Tompkins C, Curtis $M$ et al. Psychotropic drug use in longterm care facilities: A review of the literature. (ierontologist 1992;32:822833.

22. Ouslander JG, Kane RL. The cost of urinary incontinence in nursing homes. Med Carc 1984:22:69-79.

23. Ouslander JG, Fowler E. Incontinence in VA nursing home care units. J Am Geriatr Soc 1985:33:33-40.

24. Marron KR, Fillit H, Peskowitz $\mathrm{M}$ et al. The nonuse of urethral catheterization in management of urinary incontinence in the teaching nursing home. J Am Geriatr Soc 1983;31:278-281.

25. Starer P, Libow LS. Obscuring urinary incontinence: Diapering the elderly. J Am Geriatr Soc 1985;12:842-846.

26. Schnelle JF, Sowell VA, Traugher B. Reduction of urinary incontinence in nursing homes: Docs it reduce or increase costs? J Am Geriatr Soc 1988;36:34-39.

27. Ouslander JG, Kane RL, Abrass IB. Urinary Incontinence in elderly nursing home residents. JAMA 1982;248:1194-1197.

28. Michoki RJ, l.any PP. The problem of pressure sores in a nursing home population: Statistical data. J Am Geriatr Soc 1976;24:323-328.

29. Himmeistein DU, Jones AA, Woolhandler S. Hypernatremic dehydration in nursing home patients: $A \mathrm{n}$ indicator of neglect. J Am Geriatr Soc 1983.

30. Gugel RN. P'sychosocial interventions in the nursing home. In: Katz PR, Calkins E, eds. Principles and Practice of Nursing Horne Care. New York: Springer, 1989.

31. Shah BV, Barnweil GB, Beiler CS. SUDAAN; Uscr's Manual, Release 6.40. Research Triangle P'ark, NC: Rescarch Triangle Institutc, 1995

32. Phillips CD, Hawes $C$, Jannacchionne $V$ et al. Evaluating the Effects of the Resident Assessment Instrument: Methodological Issues and Approaches. Research Triangle Park, NC: Research Triangle Institute, 1995
33. Phillips (D, Morris JN, Hawes Cet al, Association of the resident assessment instrument (RAI) with changes in function, cognition, and psychosocial status. J Am Geriatr Soc, 1997:45:986-993.

34. Morris JN, Nonemaker S., Murphy $K$ et al. A commitment ws change: Revision of HCFA's RAI. J Am Geriatr Soc, 1997;45:1011-1016.

35. Sgadari A., Morris JN, Fries BE ct al. Efforts to establish reliability of the RAl. Age Aging, in press.

36. Phillips $\mathrm{CD}$, Hawes $\mathrm{C}$., Mor V ot al. Facility area variation in physical restraint use in nursing homes. Med Care, 1996:34:1149-1162.

37. Morris JN, Fries BE, Mehr D et al. MDS cognitive performance scale. I Gerontol Med Sci 1994:49:M 174-182.

38. Fries BF, Schneider D, Foley WJ et al. Retining a case mix measure for nursing homes: Resource utilization groups (RUC; III). Med Carc 1994:32:668685.

39. Analysis of the On-line Survey and Cerrification Automated Reports (OS(AR) by Vincent Mor and Jeffey Hiris, Center for Gerontelegy and Health Care Rescarch, Brown University, Providence, RI, 199.

40. Fries BF, Hawes C, Morris JN ot al. Effect of the national resident assessment instrument on selected health conditions and problems. J Am Geriatr Soc, 1997;45:994-1001.

41. Mor V, Intrater $O$, Hiris $J$ er al. Impact of the MDS on changes in nursing home discharge rates and destinations. J Am Geriatr Soc, 1997:45:10021010.

42. Buck JA. Psychotropic drug pratice in nursing homes. J Am (ieriatr Soo $1988 ; 36: 409-418$.

4.3. Sternberg J, Spector W, Drugovich $M$ et al. Use of psychoactive drugs in nursing homes: Prevalence and residents' characteristics. J Geriatr Drug Ther 1990:4:47-60.

44. Hines M, Mor V, Hawes C et al. Development of Resident Assessment Systcm and Data Base for Nursing Home Residents; Post-Implementation Telephone Survey Report. Providence, Rl: Center for Gerontology and Health Care Research, Brown University, 1994.

45. Marek KI), Rantz MJ, Fagin (Cet at. OBRA 87. Has it resulted in better quality of care? J Gerontol Nurs 1996;22:28-36.

46. Testimony before the U.S. Senate Special Committee on Aging, Medicaid Reform: Quality of Care in Nursing Homes At Risk? by Fllen T. Reap, President, Association of Health Facility Survey Agencies; John Willis, Texas State Long-Term Care Ombudsman; Scott Severens, President, National Citizens Coalition for Nursing Home Reform; William M. Russell, M.I)., Medical Director, St. Elizabeth Home, Baltimore, MD; Washingten, DC, Octoher 26, 1995. Testimony before the Democratic Policy Committec Hearing. Forum on Republican Proposals to Repeal Access to Affordable Quality Nursing Home Care, by of Elma Holder, Executive Director, National Citizens Coalition for Nursing Home Reform; Mary Fizzpatrick, family member; Morris J. Kaplan, Owner and Administrator, Ciwynedd Square Nursing (ien ter, Lansdale, PA.Washington, DC: (October 6, 1995). Testimony at a Congressional Staff and Press Briefing, Sponsored by Senators David Pryor and Jay Rockefeller and the Democratic Members of the U.S. Senate Special Committee on Aging, by Bruce Vladeck, Administrator, Health Care Financing Administration in Washington, DC (December, 199.5).

47. Phillips C.D, Hawes C, Morris JN et al. The nursing home resident assessment instrument (RAI) and the quality of assessments: The impact of the RAI on some measures of process quality. HCFA/RAI Project Working Pa per \#2, Research Triangle Institute, Research Triangle Park, NC, 1994.

48. Teno J, Branco $\mathrm{K}$, Mor $\mathrm{V}$ et al. Changes in advance care planning in nursing homes before and after the Patient Self-Determination Act: Report of a $10-$ state survey. I Am Cieriatr Soc 1997;45:939-944.

49. Rubenstein $I . Z$, Rhee $I$, Kane RL. The role of geriatric assessment units in caring for the elderly: An analytic review. J Gerontol 1982;37:513-521.

50. Applegate WB, Akins D, Vanderzwagg $R$ et al. A geriatric rehabilitation and assessment unit in a community hospital. J Am Gicriatr Soc 1983;31:206210.

51. Tulloch AJ, Moore V. A randomized controlled trial of geriatric screening and surveillance in general practice. J R Coll Cien Practitioners 1979;29:733-742.

52. Hendricksen (:, Lund E, Stromgard E. Consequences of assessment and intervention among elderly people: A threc-year randomized controlled trial. Br Med J 1984:289:1522-1524. 